\title{
COMPLETELY SEMISIMPLE RING SEMIGROUPS
}

\author{
JOHN HANNAH, J. S. RICHARDSON and JOHN ZELEZNIKOW
}

(Received 3 April; revised 4 September 1979)

Communicated by T. E. Hall

\begin{abstract}
Let $R$ be a ring in which the multiplicative semigroup is completely semisimple. If $R$ has the maximum (respectively, minimum) condition on principal multiplicative ideals. then $R$ is semiprime artinian (respectively, a direct sum of dense rings of finite-rank linear transformations of vector spaces over division rings).
\end{abstract}

1980 Mathematics subject classification (Amer. Math. Soc.): primary 20 M 25; secondary 16 A 30.16 A 32 . $16 \mathrm{~A} 42,20 \mathrm{M} 12$.

\section{Preliminaries}

The study of completely semisimple semigroups was initiated by Munn (1957). In particular, he considered various minimum conditions, showing that for the semigroups in question, the minimum conditions on principal left ideals, on principal right ideals, and on principal two-sided ideals are all equivalent (Munn (1957), Theorem 2.9). To these may be added a fourth equivalent, the minimum condition on idempotents (see below, Theorem 4). Our chief aim is the structural classification of rings in which the multiplicative semigroup is completely semisimple and satisfies the above-mentioned minimum conditions (see Theorem 4). We also show that for completely semisimple multiplicative semigroups of rings, the maximum conditions on principal two-sided ideals and on idempotents are equivalent, and again obtain a characterization of the relevant rings, in this case, as semiprime artinian rings (see Theorem 5).

Semigroups and rings will not necessarily have an identity element.

In a semigroup $S$, let

$$
E=\left\{e \in S: e^{2}=e\right\}
$$


denote the set of idempotents. Define a partial order on $E$ by: $f \leqslant e$ if and only if $f=e f e$. A nonzero idempotent $e$ is primitive in $S$ if for $f \in E, 0 \neq f \leqslant e$ implies $f=e$. We say that $S$ satisfies Min- $E$ if the minimum condition holds for $E$ under the specified order; Max- $E$ is defined dually. If $x \in S$, let

$$
J(x)=\{x\} \cup x S \cup S x \cup S x S
$$

denote the principal (two-sided) ideal generated by $x$, and

$$
I(x)=\{y \in J(x): J(y) \varsubsetneqq J(x)\}
$$

the set of nongenerators of $J(x)$. Then $S$ is called completely semisimple (see Clifford and Preston (1967), p. 32) if for each nonzero $x \in S$, the Rees quotient semigroup $J(x) / I(x)$ contains a primitive idempotent; when this is the case, it is well known that every nonzero idempotent of $J(x) / I(x)$ is primitive (for example, see Clifford and Preston (1961), Exercise 11, p. 84). We let Min- $J$ signify the minimum condition on the set of principal ideals of $S$ (ordered by inclusion); Max- $J$ is its dual.

A ring or semigroup $S$ is regular if for all $x \in S$, there exists $y \in S$ with $x y x=x$, or equivalently, if each principal right ideal is generated by an idempotent. We call a semigroup $S$ with identity directly finite if for $x, y \in S, x y=1$ implies $y x=1$; for rings, this agrees with the usual definition.

A ring is semiprime if it contains no nonzero nilpotent (one-sided) ideals, and artinian if it satisfies the minimum condition on right ideals. Following Petrich (1974), p. 69, we shall call a ring atomic if it is a (direct) sum of minimal right ideals.

\section{Results}

Proposition 1. For a semigroup $S$ with set of idempotents $E$, the following are equivalent:

(a) $S$ is completely semisimple;

(b) $S$ is regular and for $e, f \in E, f<e$ implies $J(f) \mp J(e)$;

(c) $S$ is regular and eSe is directly finite for each $e \in E$.

Proof. (a) $\Rightarrow$ (b) Completely semisimple semigroups are regular by Clifford and Preston (1967), 6.48 (see 2.51). If $f<e$, certainly $J(f) \subseteq J(e)$; moreover, by (a) $e$ is primitive in $J(e) / I(e)$, so $f$ is the zero thereof, whence $f \in I(e)$ and $J(f) \mp J(e)$.

(b) $\Rightarrow$ (c) Let $x, y \in e S e$ with $x y=e$. Put $f=y x$; then $x f y=e$ and $y e x=f$, so $J(f)=J(e)$. Further, $f=f^{2} \leqslant e$, so $y x=f=e$ by (b).

(c) $\Rightarrow$ (a) Let $x \in S-\{0\}$. By regularity, $J(x)=J(e)$ for some $e \in E-\{0\}$. Suppose $f=f^{2} \leqslant e$ with $J(f)=J(e)$. Then $e=a f b$ for some $a, b \in S$; since $e=e a f e b e$, we may assume $a f, b \in e S e$. By (c), baf $=e$, whence $e=e f=f$. Thus $e$ is primitive in $J(x) / I(x)$. 
Note that for rings, (c) characterizes the multiplicative concept of complete semisimplicity in entirely ring-theoretic terms. For rings with identity, there is a simpler characterization:

COROLlary 2. Let $R$ be a ring with identity. Then the multiplicatice semigroup of $R$ is completely semisimple if and only if. $R$ is regular and directly finite.

Proof. It remains to show that if $R$ is directly finite and $e=e^{2} \in R$, then $e R e$ is directly finite. Let $x, y \in e R e$ with $x y=e$. Then

$$
(x+1-e)(y+1-e)=x y+1-e=1,
$$

so

$$
1=(y+1-e)(x+1-e)=y x+1-e,
$$

whence $y x=e$.

LemMA 3. Let $R$ be a ring and $E$ its set of multiplicatile idempotents.

(a) Max-E implies Min-E.

(b) If $R$ has an identity element, Min- $E$ implies Max-E.

(c) Suppose Min-E holds. Then every nonzero idempotent is a sum of primitice idempotents.

ProOF. (a) If $e_{1}>e_{2}>e_{3}>\ldots$, then $e_{1}-e_{2}<e_{1}-e_{3}<\ldots$.

(b) If $e_{1}<e_{2}<\ldots$, then $1-e_{1}>1-e_{2}>\ldots$.

(c) Assume the contrary, and choose $e \in E-\{0\}$ minimal with respect to not being such a sum. Now $e$ cannot be primitive: say $0 \neq f=f^{2}<e$. Then also $0 \neq e-f=(e-f)^{2}<e$, so both $f$ and $e-f$ are sums of primitive idempotents (by the minimality of $e$ ). But $e=f+(e-f)$, a contradiction.

THEOREM 4. For a semigroup $S$, the following are equiralent:

(a) $S$ is completely semisimple and satisfies Min- $J$;

(b) $S$ is completely semisimple and satisfies Min-E;

(c) $S$ is regular and satisfies Min- $E$.

Furthermore, if $S$ is the multiplicative semigroup of a ring $R$, then (a), (b) and (c) are equivalent to each of the following conditions:

(d) $R$ is semiprime atomic;

(e) $R$ is a direct sum of dense rings of finite-rank linear transformations of tector spaces over division rings. 
PrOOF. (a) $\Rightarrow$ (b) $\Rightarrow$ (c) by Proposition 1 .

(c) $\Rightarrow$ (a) Let $x \in S$. By regularity, the set of idempotents in $J(x)-I(x)$ is nonempty. By Min- $E$, this set contains a minimal element, which gives a primitive idempotent in $J(x) / I(x)$. Thus $S$ is completely semisimple. It is an immediate consequence of Hall (1970), Theorem 1, that for regular semigroups, Min- $E$ implies Min- $J$.

Now let $R$ be a ring with multiplicative semigroup $S$.

(c) $\Rightarrow$ (d) If $0 \neq x \in R$, then by regularity and Lemma 3(c),

$$
x R \leqslant e_{1} R+\ldots+e_{n} R
$$

for primitive idempotents $\epsilon_{i}$. By regularity again, each $e_{i} R$ is minimal (Clifford and Preston (1967), 6.38). Hence $R$ is a sum of minimal right ideals, and therefore (Jacobson (1956), Theorem 1, p. 61) a direct sum of such ideals, that is, atomic. Regularity implies that $R$ is semiprime.

(d) $\Rightarrow$ (e) Since $R$ is atomic, it is its own right socle, and thus the direct sum of its homogeneous components, each of which is a simple ring as $R$ is semiprime (Jacobson (1956), p. 64-65). Now (e) follows from McCoy (1964), 7.13.

(e) $\Rightarrow$ (c) It is easy to reduce to the case where $R$ is a single dense ring of finite-rank linear transformations of a vector space $V_{D}$ over a division ring $D$. Such an $R$ is regular (McCoy (1964), 7.9). Moreover, if $f=f^{2}<e=e^{2} \in R$, then $f V \varsubsetneqq e V$, for $f \leqslant e$ and $f V=e V$ imply

$$
(e-f) V=(e-f) e V=(e-f) f V=0 .
$$

Since $e V$ is finite-dimensional over $D$, it follows that $R$ satisfies Min- $E$.

Remarks. Theorem 4 extends the characterization of semiprime atomic rings given in Petrich (1974), II.6.1, a result in which the equivalence of (d) and (e) above-no doubt well known - - is implicit (see also Petrich (1974), II.2.8). Note also that the equivalent conditions (a), (b) and (c) imply that $S$ is regular with Min- $J$, but even for rings with identity the converse does not hold. For the full ring of linear transformations of an infinite-dimensional vector space is regular (McCoy (1964), 7.3) with Min-J (Petrich (1974), I.4.2), but does not satisfy Min-E, since the projections onto an infinite descending chain of subspaces give rise to an infinite descending chain of idempotents. A second instance of the same situation is provided by Example 8 below.

THEOREM 5. For a semiyroup $S$, each of the following conditions implies the next:

(a) $S$ is completely semisimple and satisfies $\operatorname{Max}-J$;

(b) $S$ is completely semisimple and satisfies Max-E;

(c) $S$ is regular and satisfies Max-E. 
Furthermore, if $S$ is the multiplicative semigroup of a ring $R$, then (a), (b) and (c) are equivalent to each other and to:

(d) $R$ is semiprime artinian, that is, a finite direct sum of full matrix rings over division rings.

Proof. (a) $\Rightarrow$ (b) $\Rightarrow$ (c) by Proposition 1 .

Now let $R$ be a ring with multiplicative semigroup $S$.

(c) $\Rightarrow$ (d) Suppose that $R$ is regular with Max-E. We first show that $R$ has an identity element. Let $e$ be a maximal idempotent in $R$, and let $x \in R$. We apply a familiar argument of von Neumann (1936). Since $R$ is regular, $x R=f R$ for some $f=f^{2} \in R$; then

$$
e R+x R=e R+f R=e R+(f-\ell f) R .
$$

Again, $(f-e f) R=g R$ where $g=g^{2} \in R$, and $e g \in e(f-e f) R=0$. Let $h=e+g-g e$. Since $h e=e$ and $h g=g$, we have

$$
e R+x R=e R+g R=h R .
$$

Moreover, $h=h^{2} \geqslant e$, so $h=e$ by choice of $e$. Hence $x \in e R$, and $e x=x$. Similarly $x e=e$, so $e$ is the required identity element.

By Lemma 3(a), $R$ has Min- $E$. Thus by Theorem $4, R$ is semiprime atomic, and in particular, a direct sum of minimal right ideals.

Since $R$ has an identity element, this sum must be finitc. Hence $R$ is artinian. The Artin-Wedderburn Theorem (Jacobson (1956), p. 40; see also Corollary 1, p. 39) now applies.

(d) $\Rightarrow$ (a) It follows from Theorem 4 that (d) implies the complete semisimplicity of $S$. A matrix ring over a division ring satisfies Max- $J$ by Petrich (1974), I.3.6, and extension to a direct sum of such matrix rings is straightforward.

We conclude with four examples to show what can go wrong if we relax some of the hypotheses used above.

EXAMPLE 6. The bicyclic semigroup

$$
\langle p, q: p q=1 \neq q p\rangle
$$

is regular and satisfies Max- $E$ but not Min- $E$ (Clifford and Preston (1961), 2.53). Moreover, it is not completely semisimple (for example, by Proposition 1), so in Theorem 5 we see that (c) does not imply (b) for non-ring-semigroups.

EXAmPLE 7. The implication (b) $\Rightarrow$ (a) also breaks down for general semigroups. To see this consider the following construction. Let $C_{2}, C_{3}, C_{4}, \ldots$ be the semilattices 
which are chains of lengths $2,3,4, \ldots$ (respectively) and assume that these chains have a common zero element, 0 say. Now let $E$ be the 0 -direct union of $C_{2}, C_{3}, C_{4}, \ldots$ (see Clifford and Preston (1967). Section 6.3) so that $E$ is a semilattice satisfying Max-E and Min- $E$. Then $T_{E}$, the Munn semigroup of $E$ (see Howie (1976), Section V.4), is an inverse semigroup with $E$ as its semilattice of all idempotents (so that, by Theorem 4, $T_{E}$ is completely semisimple) but it does not satisfy Max- $J$.

The remaining examples are taken from the simple right self-injective rings studied by Goodearl and Handelman (1975). Firstly we reinterpret the principal multiplicative ideals in a regular ring.

LEMMA 8. Let $a, b \in R$, a reyular ring. Then $J(a) \subseteq J(b)$ if and only if there is a one-toone right $R$-module homomorphism from aR to $b R$.

PROOF. Since $R$ is regulur we may assume that $a$ and $b$ are idempotents. If $J(a) \subseteq J(b)$, say $a=x b y$, then $\varphi: a R \rightarrow b R$ given by $\varphi(a r)=b y a r$ is an $R$-module homomorphism which is one-to-one since if byar = byas then

$$
a r=a^{2} r=x b y a r=x b y a s=a s .
$$

Conversely, suppose $\varphi: a R \rightarrow b R$ is an $R$-monomorphism, with $\varphi(a)=b x$. There exists $y \in R$ with $b x=b x y h x$, and we have

$$
\varphi(a y b x)=\varphi(a) y b x=b x=\varphi(a) .
$$

Since $\varphi$ is one-to-one, $a=a y b x \in J(b)$.

Following Goodearl and Handelman we shall write $a R \leqq b R$ if there is a one-toone $R$-module homomorphism $\varphi: a R \rightarrow b R$.

EXAMPLE 9. There is a regular ring $R$ with identity which has only two principal multiplicative ideals but does not satisfy Max- $E$ or Min- $E$.

For let $R$ be a simple right self-injective ring which is not directly finite (see Goodearl and Handelman (1975), Example (a), p. 805); then $R$ is regular (Goodearl and Handelman (1975), Remark, p. 801). The only principal multiplicative ideals of $R$ are 0 and $R$. To see this, let $0 \neq a \in R$. Then $R \lesssim a R$ (Goodearl and Handelman (1975), Theorem 2.1, proof that (d) implies (a)), so by Lemma 8 we have $R=J(1) \subseteq J(a)$. On the other hand, as $R$ is not directly finite it contains a copy of the bicyclic semigroup, so does not satisfy Min-E. By Lemma 3(a) it does not satisfy $M a x-E$ either.

Thus without complete semisimplicity even the strongest possible chain condition on principal multiplicative ideals fails to force any chain condition on idempotents.

Example 10. There is a ring $R$ with identity whose multiplicative semigroup is completely semisimple but does not satisfy Max- $J$, Min- $J$, Max- $E$ or Min-E. 
For let $R$ be a simple right self-injective ring which is directly finite but not artinian (Goodearl and Handelman (1975), Example (b), p. 805); as before. $R$ is regular. By Corollary 2, the multiplicative semigroup of $R$ is completely semisimple. Thus $R$ does not satisfy Max- $E$ or Min- $E$ (since it is not atomic). Indeed $R$ does not have any primitive idempotents: if it did, the socle of $R$ would be nonzero so all of $R$ (as $R$ is simple); but then $R$ would be artinian.

We determine the principal multiplicative ideals of $R$ as follows. Let $D$ be the "dimension function" on the lattice of principal right ideals of $R$ (Goodearl and Handelman (1975), Theorem 3.17). If $a \in R$, then we claim that

$$
J(a)=\{x \in R: D(x R) \leqslant D(a R)\} .
$$

Indeed, by Lemma 8 we know that $x \in J(a)$ if and only if $x \leqq a R$, and this last is equivalent to $D(x R) \leqslant D(a R)$ (Goodearl and Handelman (1975), Theorem 3.17(b)). Since the range of $D$ is the entire unit interval $[0,1]$ (Goodearl and Handelman (1975), Theorem 3.17(d)), it follows that the lattice of principal multiplicative ideals of $R$, ordered by inclusion, is isomorphic to $[0,1]$; so, in particular, $R$ does not satisfy Max- $J$ or Min- $J$.

\section{References}

A. H. Clifford and G. B. Preston (1961 and 1967), The algebraic therry of semigroups, Vols 1 and II (American Mathematical Society, Providence, Rhode Island).

K. R. Goodearl and D. Handelman (1975), 'Simple self-injectıve rings'. Comm. Algebra 3 (9), 797834.

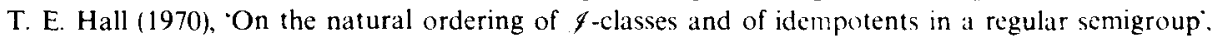
Glasgow Math. J. 11, 167-168.

J. M. Howie (1976), An introduction to semigroup theory (London Math. Soc. Monographs 7, Academic Press, London).

Nathan Jacobson (1956), Structure of rings (American Mathematical Society, Providence. Rhode Island). N. H. McCoy (1964), The theory of rings (Macmillan, New York).

W. D. Munn (1957), 'Semigroups satisfying minimal conditions', Proc Glasgow .Hath. Assoc. 3. 145152. Mario Petrich (1974), Rings and semigroups (Lecture Notes in Mathemattics 380, Springer-Verlag. Berlin. Heidelberg, New York).

J. von Neumann (1936), 'On regular rings', Proc. Nat. Acid. Sci. L.S.A. 22, 707713.

Department of Mathematics

University of Melbourne

Parkville, Australia 3052
Department of Mathematics University of Melbourne Parkville, Australia 3052

Department of Mathematical Sciences

Northern Illinois University at De Kalb

Illinois 60115

USA 